\title{
Largely Enhancing Luminous Efficacy, Color- Conversion Efficiency, and Stability for Quantum Dot White LEDs Using the Two-dimensional Hexagonal Pore Structure of SBA-15 Mesoporous Particles
}

Jiasheng Li, ${ }^{1,2}$ Yong TANG, ${ }^{1}$ Zongtao Li, ${ }^{1,2},{ }^{*}$ XinRui Ding, ${ }^{1, *}$ Binhai YU, ${ }^{1}$ AND LIWEI LIN ${ }^{3}$

${ }^{1}$ Engineering Research Center of Green Manufacturing for Energy-Saving and New-Energy Technology, South China University of Technology, Guangdong, 510640, China

${ }^{2}$ Foshan Nationstar Optoelectronics Company Ltd., Foshan 528000, China

${ }^{3}$ Department of Mechanical Engineering, University of California, Berkeley, CA 94720-5800,

USA

* $\underline{\text { meztli@scut.edu.cn }}$

*ingxr@scut.edu.cn 


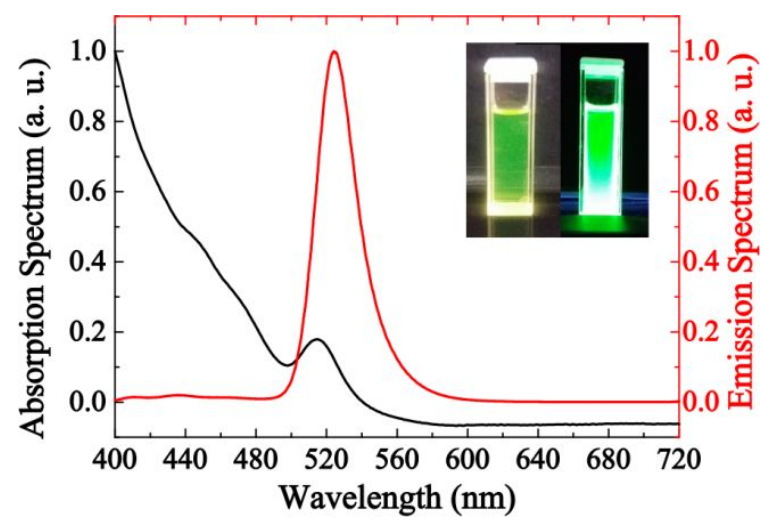

Fig. S1. The absorption and emission spectra of QDs.

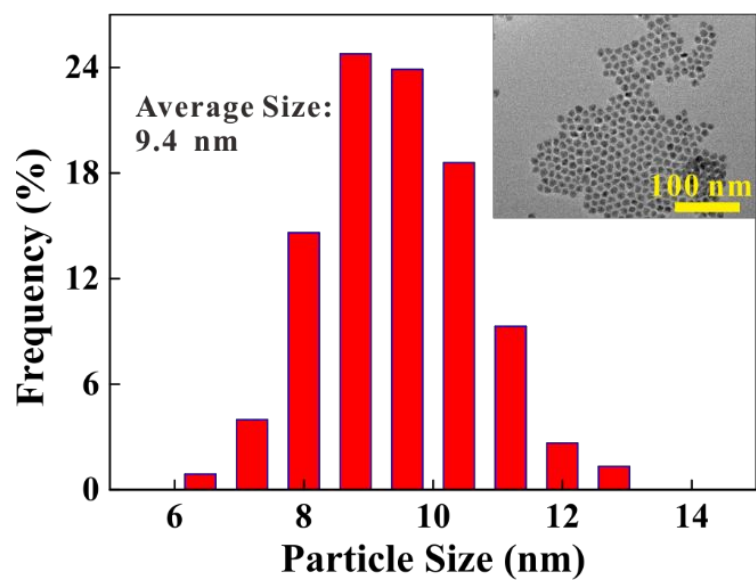

Fig. S2 The particle size distribution of QDs.
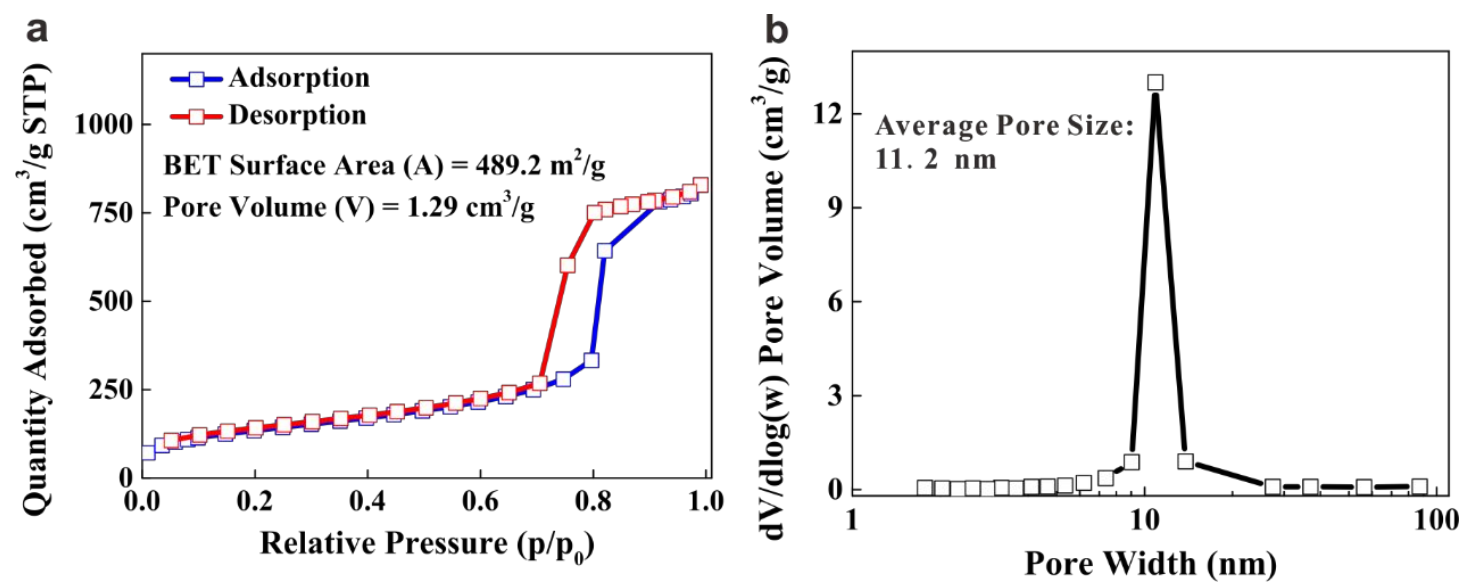

Fig. S3. The (a) adsorption-desorption isotherm and (b) pore size distribution of SBA-15 MPs. 
a

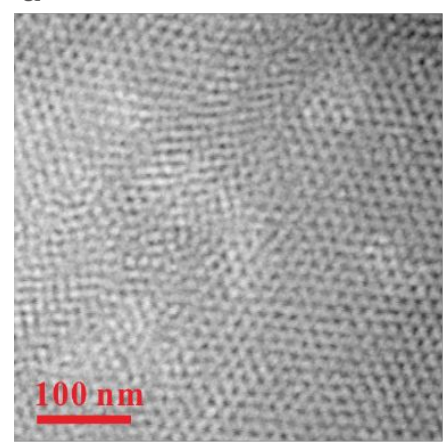

d

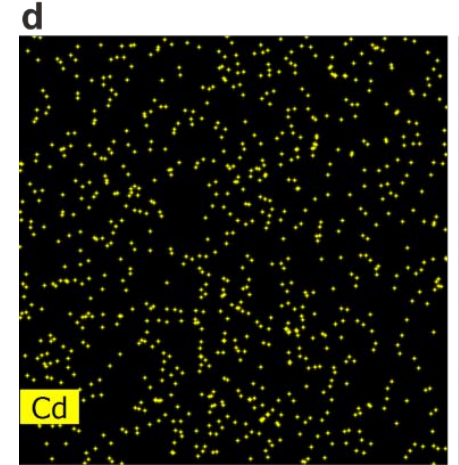

b
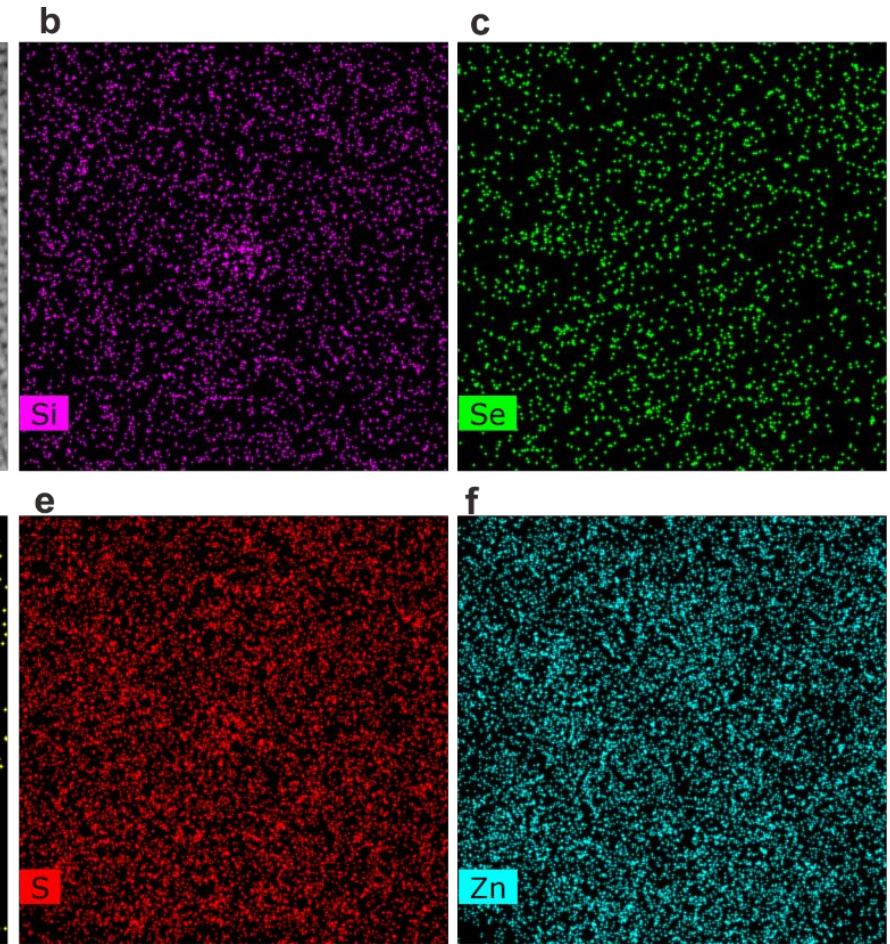

Fig. S4 The (a) top-viewed STEM image of the SBA-15 MP/QD hybrid particles. (b)-(f) Elemental Si, Se, Cd, S, and Zn mapping images of the hybrid particles, respectively, collected from the same location of (a).

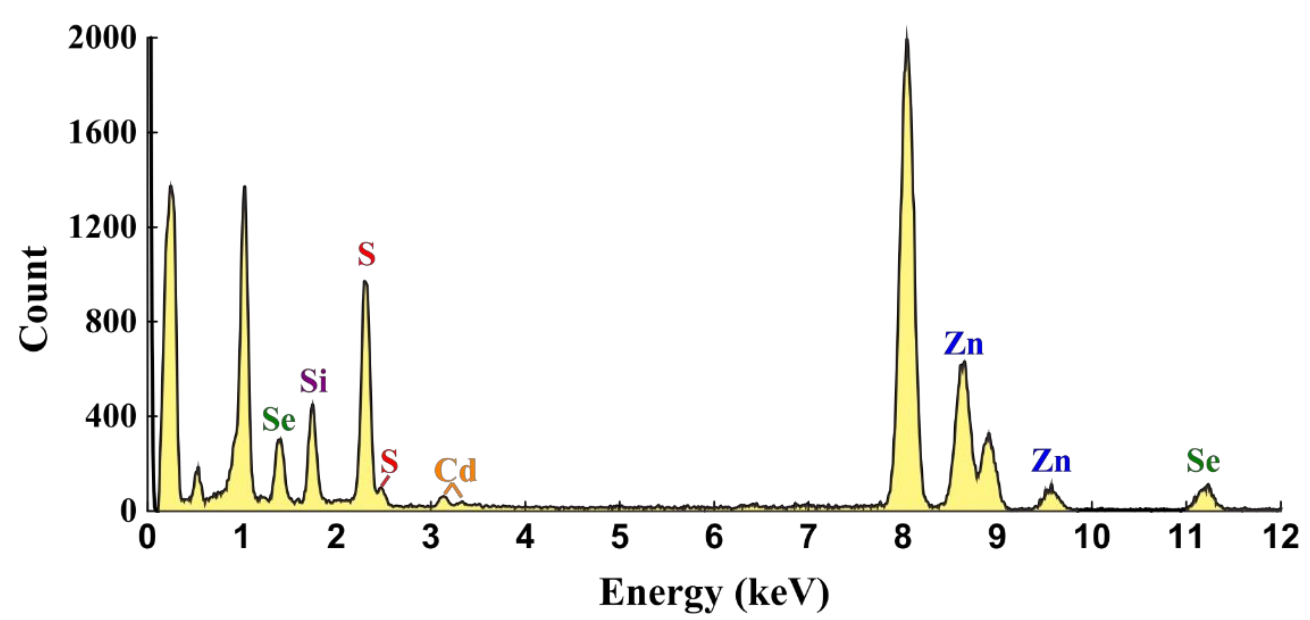

Fig. S5 EDS spectra of SBA-15 MP/QD hybrid particles given in Fig. S4. 
a
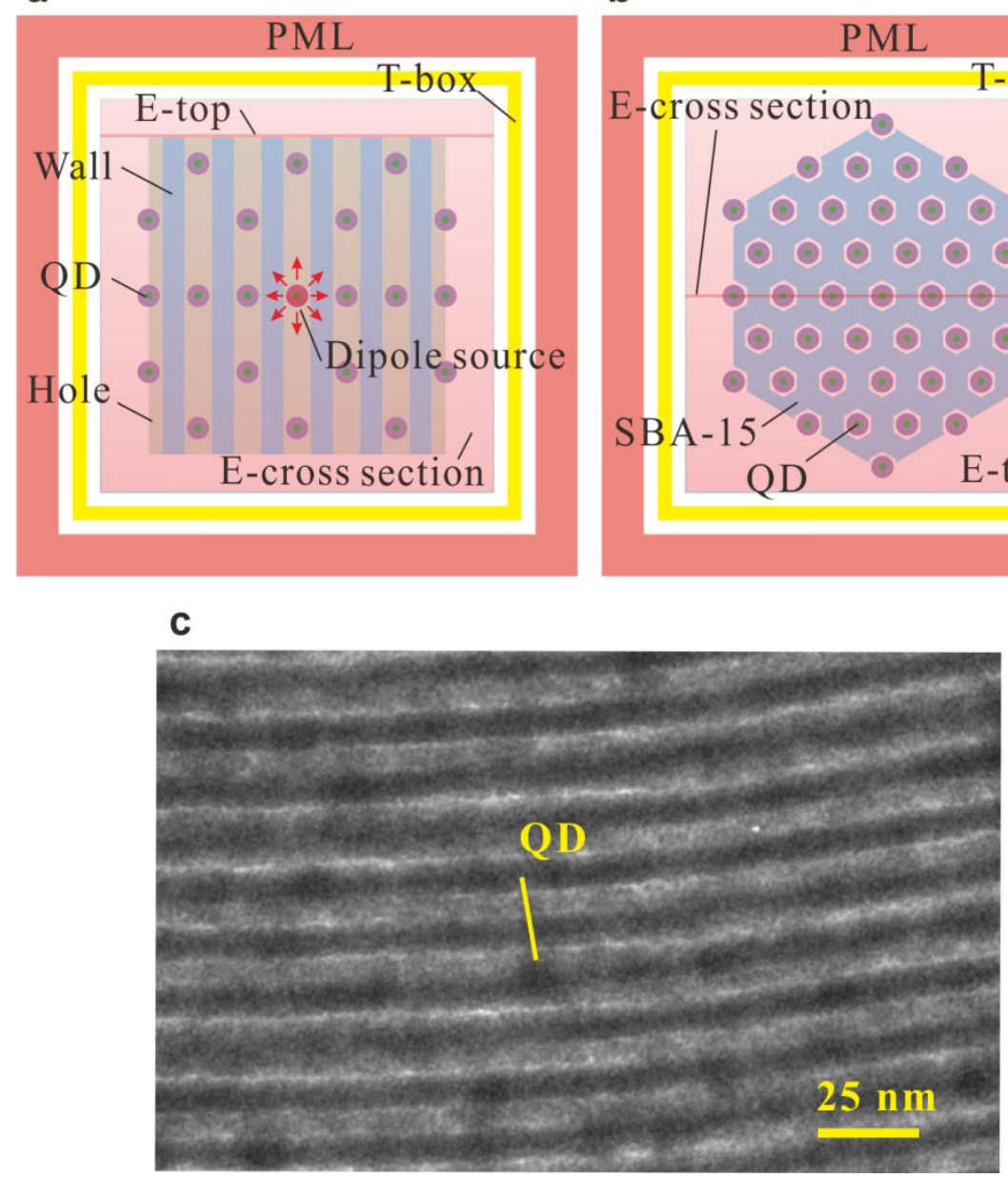

b

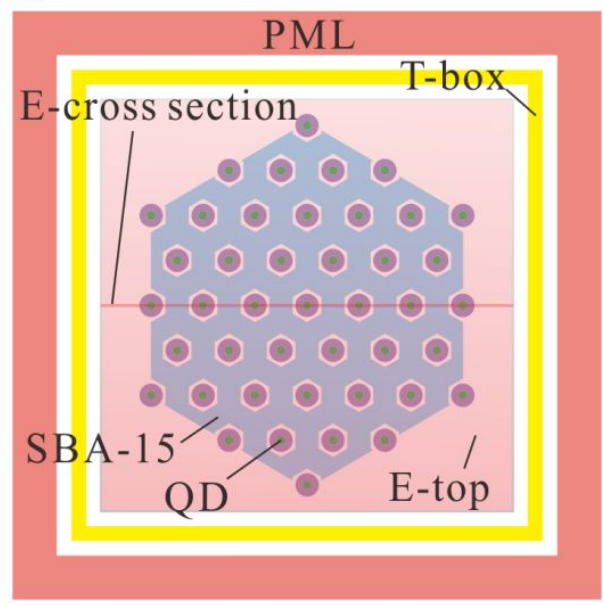


wavelength is $520 \mathrm{~nm}$. The background material of the free region is the silicone. The complex refractive index of the Si-based SBA-15 particle and CdSe/ZnS QDs are set according to literatures ${ }^{2}$. Considering the harsh calculation requirements, the FDTD calculation region is set with a size of $150 \times 150 \times 350 \mathrm{~nm}$, and a simplified hybrid particle with 43 two-dimensional hexagonal pores is established; the particle size of this hybrid particle is approximately $1 / 3$ times of that shown in Figure 2(d). According to the TEM image shown in Figure 2(b), the hole and wall of the SBA-15 are set with sizes of $10 \mathrm{~nm}$ and $5 \mathrm{~nm}$, respectively. Furthermore, 1 2 QDs are located in one hexagonal pore per hundred nanometer shown in Figure S2(c). It is assumed that QDs with high concentration are uniformed dispersed in the SBA-15 particle, each hole is with 3 QDs for covering the top, middle, and bottom region, totally $129 \mathrm{CdSe} / \mathrm{ZnS}$ QDs with size of $9 \mathrm{~nm}$ are placed inside the hole of SBA-15 particle. The maximum mesh size is $0.05 \mathrm{~nm}$ to ensure the simulation accuracy and the simulation time is set large enough to ensure energy convergence $(<$ $0.001 \%)$.

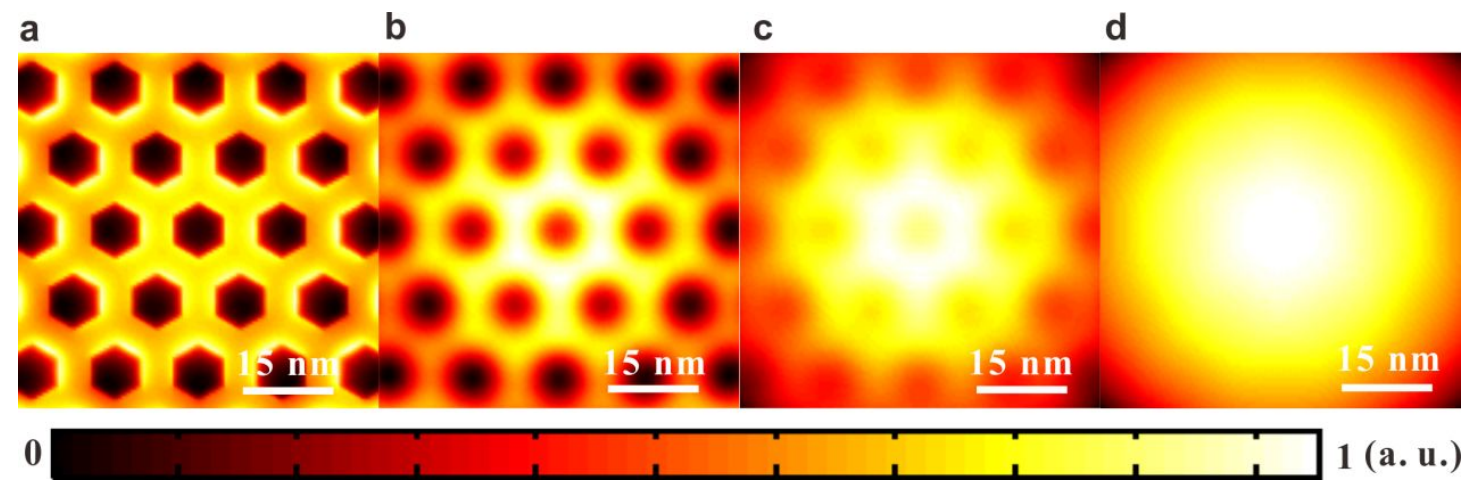

Fig. S7. The electromagnetic field of escaping light at locations with different distance from the top surface of a MP/QD hybrid particle. The electromagnetic field is (a) $0 \mathrm{~nm}$, (b) $5 \mathrm{~nm}$, (c) $8 \mathrm{~nm}$, and (d) $12 \mathrm{~nm}$ away from the top surface of the hybrid particle. 


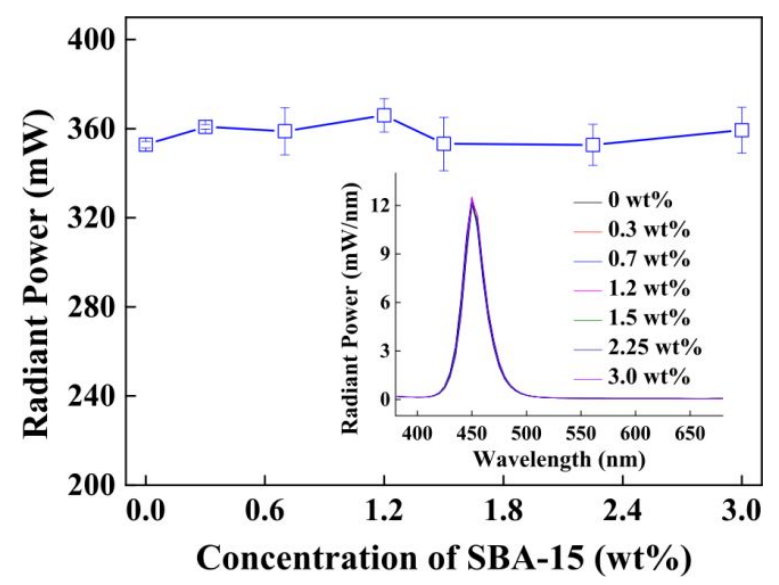

Fig. S8. The radiant power of LEDs using MP-only composites with various MP concentrations.

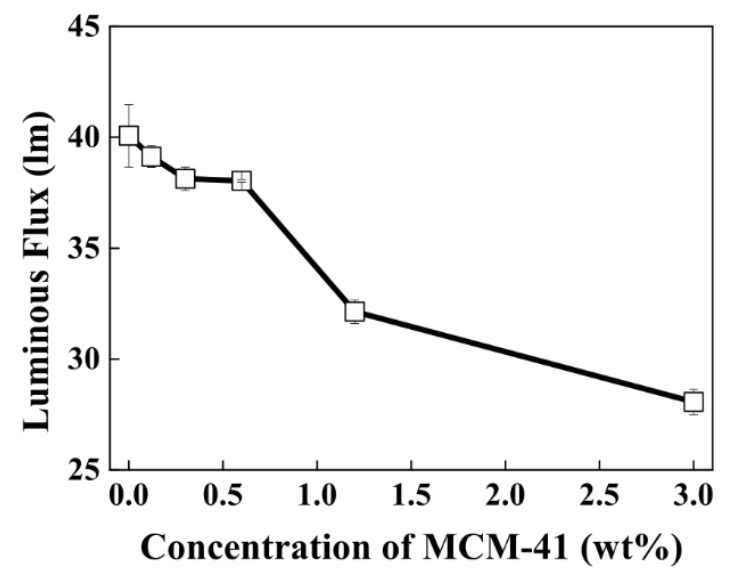

Fig. S9. The luminous flux of LEDs using MP/QD hybrid composites of various MCM-41 concentrations; the QD concentration is $0.3 \mathrm{wt} \%$. 

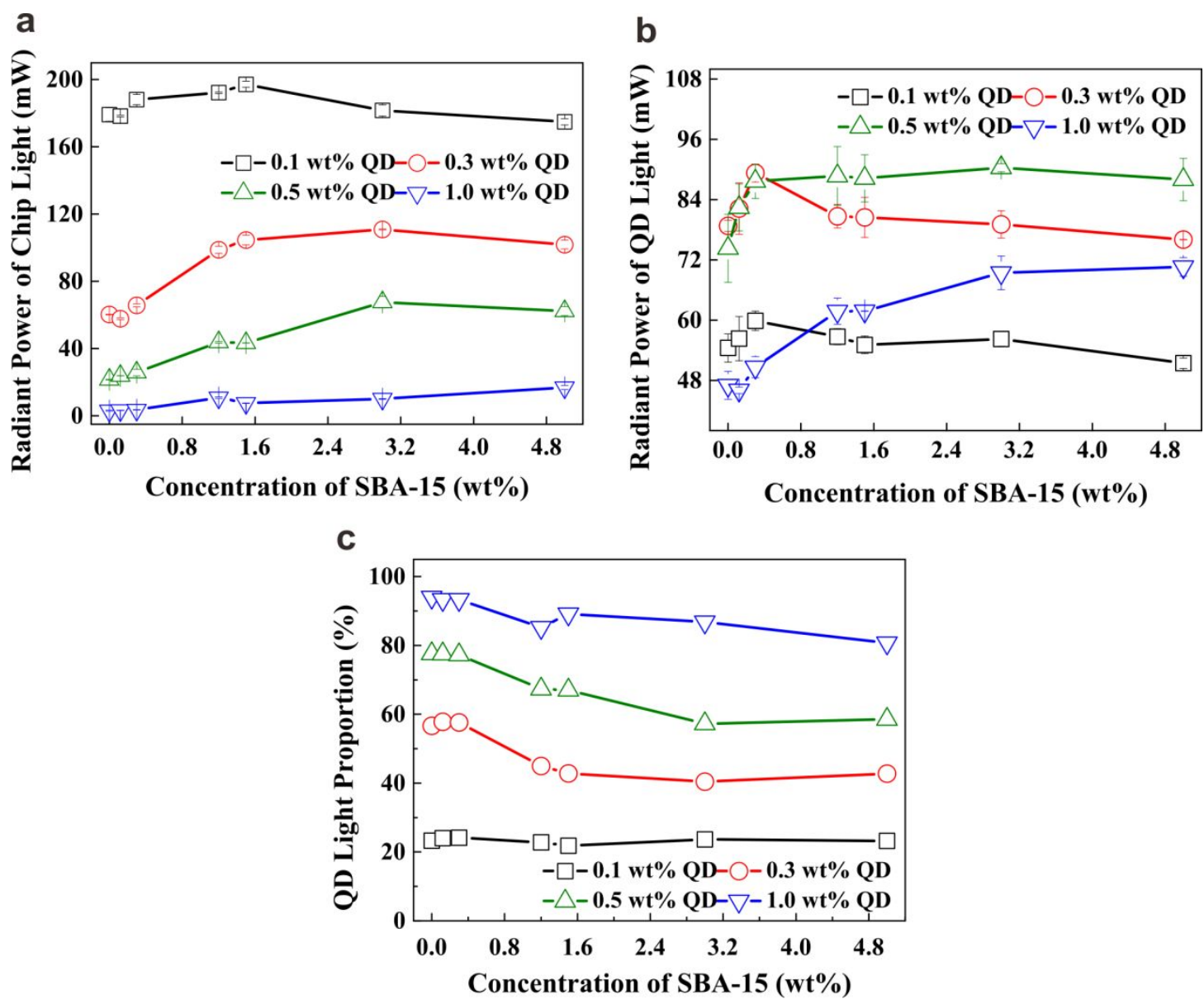

Fig. S10. The (a) radiant power of chip light, (b) radiant power of QD light, and (c) QD light proportion (ratio of radiant power of QD light to that of the total light) for LEDs using MP/QD hybrid composites of various concentrations.

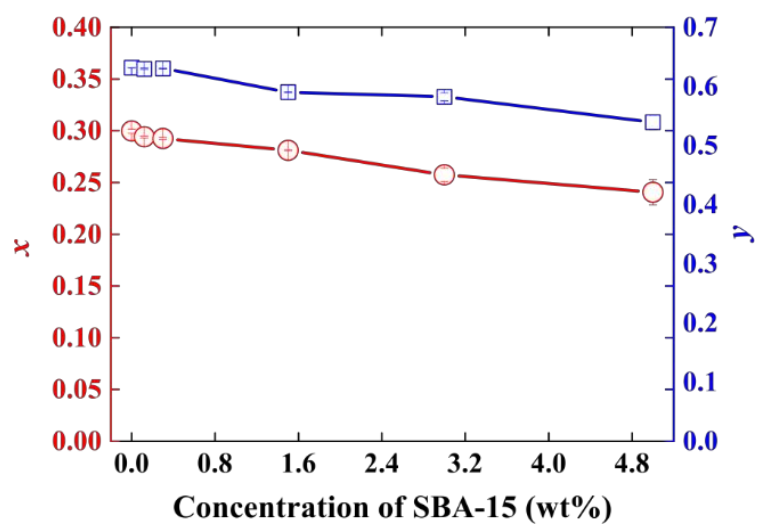

Fig. S11. The CIE 1931 coordinates of white LEDs with QD concentration of $1 \mathrm{wt} \%$ and SBA-15 MPs of various concentration. 
a

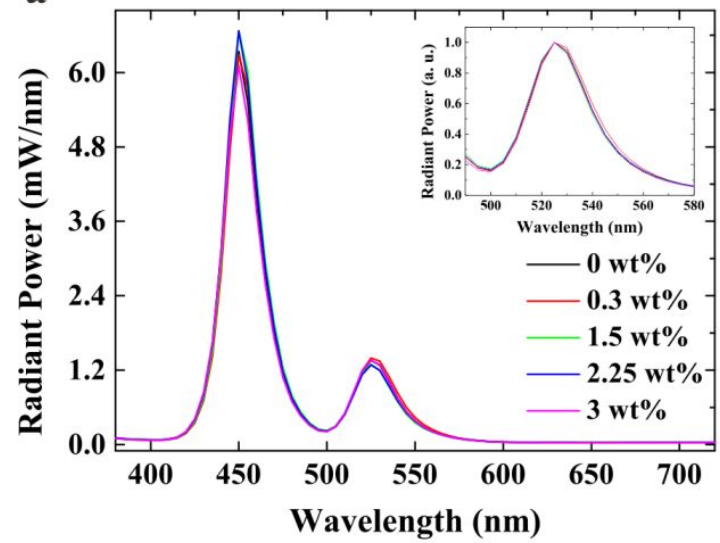

c

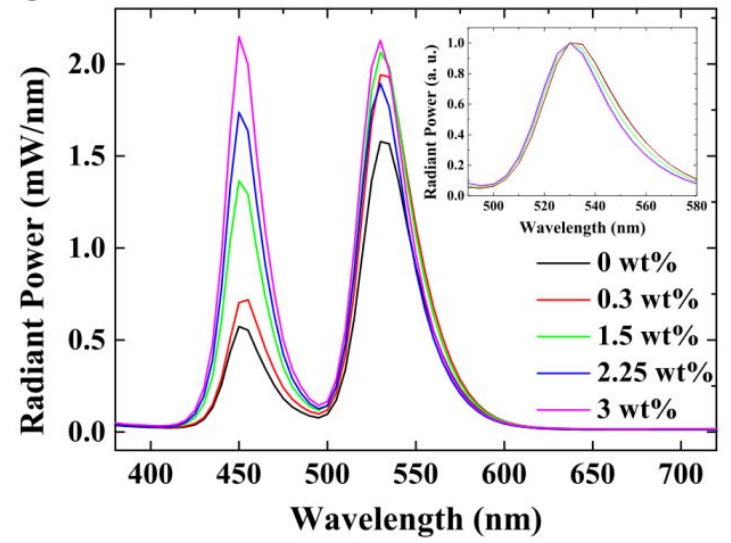

b

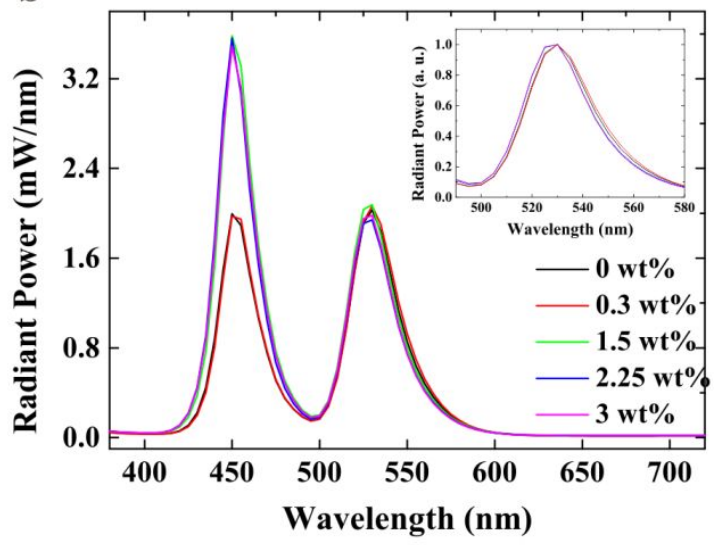

d

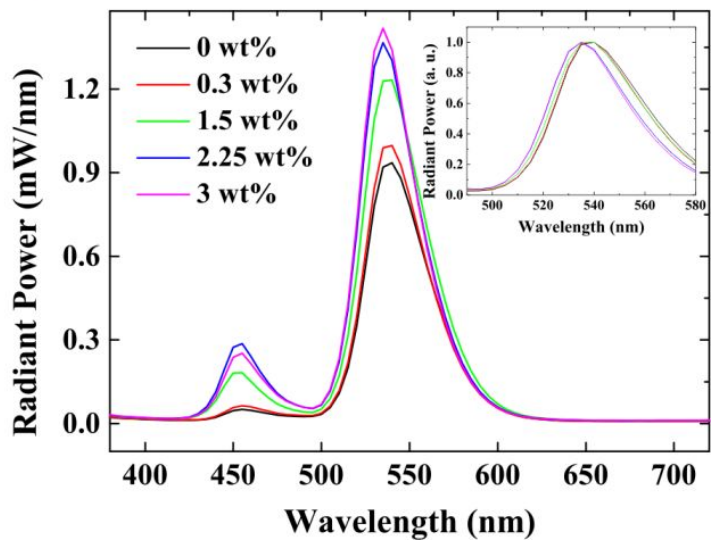

Fig. S12. The spectra of LEDs using MP/QD hybrid composites with various concentrations. The concentrations of the QDs are (a) $0.1 \mathrm{wt} \%$, (b) $0.3 \mathrm{wt} \%$, (c) $0.5 \mathrm{wt} \%$, and (d) $1.0 \mathrm{wt} \%$.

\section{References}

1. Yee, K. S. Numerical solution of initial boundary value problems involving Maxwell's equations in isotropic media. IEEE Trans. Antennas Propag 1966, 14, 302.

2. Palik, E. D. Handbook of Optical Constants of Solids. Academic Press: San Diego, CA, USA, 1998, volume 3. 